\title{
CONCEPTION AND PARAMETRIC DESIGN WORKFLOW FOR A TIMBER LARGE-SPANNED REVERSIBLE GRID SHELL TO SHELTER THE ARCHAEOLOGICAL SITE OF THE ROMAN SHIPWRECKS IN PISA
}

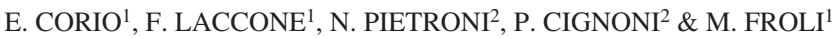 \\ ${ }^{1}$ DESTeC, School of Engineering, University of Pisa, Pisa, Italy. \\ ${ }^{2}$ ISTI, CNR, Pisa, Italy.
}

\begin{abstract}
Reciprocal structures or nexorade are composed by the assembling of groups of three or more beams mutually connected by mono-lateral $\mathrm{T}$ joints in a way that any relative movement is suppressed. This kind of structures can be easily built in relatively unprepared sites, dismantled, transported and re-used even by not specialized handcraft. For these reasons, reciprocal structures have been widely used in the past for military purposes, and nowadays they seem to satisfy very well the different requirements of a quick and temporary shelter of a large archaeological area when they are shaped as grid shells.

This paper proposes the design of a reversible, reciprocal framed grid shell to shelter the archaeological site of the Roman Shipwrecks in Pisa. The structure must protect excavations and archaeologists from the weather and provide an easy access to visitors. Additionally, it must allow for easy disassembling and moving to another site.

The design choices aim at optimizing both structural efficiency and esthetical qualities. A parametric workflow for both the form finding and the digital fabrication processes has been developed, and a prototype of accommodative steel T-joint for timber reciprocal beams has been realized. Finally, a model using CNC-cutting tested the structural feasibility of such a design approach.

Keywords: archeological shelters, cardan joints, digital fabrication, form finding, parametric design, reciprocal frame, reversible structures, timber grid shells.
\end{abstract}

\section{RECIPROCAL FRAMES}

\subsection{History}

The principle of reciprocity in structural design, i.e. a system consisting of beams mutually connected to simple mono-lateral joints, has been used since the antiquity. In the first archetypes, such as Eskimo tents or Indian tepees, the shortage of local building material dictated the idea of enclosing spaces with relatively small, mutually supporting elements. Throughout the centuries, some famous architects and engineers such as Villard de Honnecourt in the Middle Ages, and Leonardo da Vinci and Sebastiano Serlio in the Renaissance, studied the possibility of spanning distances longer than the available timber beams [1].

After being neglected for more than two hundred years, reciprocal frame (RF) system was practically applied to architecture at the beginning of 1900. During the reconstruction era following the first world war, Zollinger developed the lamella roof framing system, a successful variant on the tectonics of the timber nexorade [2]. In the last decades, several reciprocal framed timber structures have been built, mainly in Japan and in the UK. 


\subsection{State of the art}

Nowadays, timber nexorade has again become a topic for academic research and experimental structures, such as the Forest Park temporary pavilion by Shigeru Ban and ARUP AGU, the Serpentine Pavilion by Balmond and Siza, and the research pavilion by Nabaei and Weinand [3]. These projects, using different element sections, joints and patterns, highlighted a renewed interest in the geometry and structural behavior of a building system that should still be further investigated.

Since the parameters that generate the RF system induce strong geometrical constrains, the design of this kind of structures requires a form-finding step. Baverel et al. proposed two different methods to transform an initial elementary spatial configuration into a nexorade. The first method uses genetic algorithm, and is based on a geometrical optimization [4]; the second method uses a dynamic relaxation algorithm, and introduces a fictitious mechanical behavior [5]. Kohlhammer and Kotnik [6, 7] described the systemic behavior of a plane reciprocal frame structure, and presented a general design and analysis method. Song et al. [8], observing the RF self-similar and highly symmetric patterns, established a duality with planar tiling theory. Once the RF-tessellation is formed on the plane, it can be regarded as a flat polygonal mesh, where the junctions between the rods are the mesh vertices. The planar mesh is then lifted to the $3 \mathrm{D}$ surface employing a conformal map that preserves the angles between the edges.

A careful joint design is important for improving the structural behavior and achieving an easy erection and dismantlement of RF structures. Elements can generally be joined without mechanical connections, relying only on pressure and friction. In larger structures, the frictional forces can be supported by simple connecting techniques, such as tying together or notching of the bars [1].

Studying the structural behavior of nexorade, researchers highlighted many interesting phenomena that characterize this system. A strong link was proved to exist between geometric properties and bending stiffness of the RF system, but there are actually no practical formulas quantifying this influence.

\subsection{Our contribution}

Due to the lack of computational support to design and construct RF-unit-based structures, most existing realizations are restricted to small structures based on quadrilateral cells. Extending the RF system to the design of large-spanned grid shells while maintaining tectonic simplicity is a challenging task.

In this paper, we describe a parametric design workflow developed within Grasshopper [9], a generative modelling plug-in of Rhinoceros ${ }^{\circledR}$ [10]. Instead of starting from a planar RF pattern and mapping it with an aim 3D surface, we started from an elementary 3D configuration with multi-connected joints, and transformed it into a spatial RF grid with mono-lateral joints. First, we transformed the initial surface into a regular, symmetric mesh with the desired edge length, then we extracted the mesh edges and geometrically manipulated them to obtain the RF grid. Starting from a triangular mesh, we found a tri-hex, Wien-thatch-like spatial pattern whose triangular-shaped corner element particularly improved the structural stiffness compared to their quadrilateral competitors.

In order to verify the structural feasibility of the shell, we tested the load bearing capacity and the global buckling, which is the most common collapse mechanism of large-spanned grid-shells. The structural evaluation was carried out using Karamba® [11]. Since the 
traditional connections used for timber nexors couldn't carry loads acting on this structure, a tailored-made steel $\mathrm{T}$ joint has been designed.

\section{CONCEPTION OF THE ARCHEOLOGICAL SHELTER}

\subsection{Requirements}

This paper proposes the design of a reversible, reciprocal framed grid shell to shelter the archeological site of the Roman Shipwrecks in Pisa. The site is very close to the famous Leaning Pisa Tower, just outside the city wall. Since 1998, more than eleven wooden ships have been excavated (Fig. 1). The discovery of this underground treasure, preserved thanks to the clayey ground and the lack of oxygen, is an extraordinary event from an archeological and scientific point of view. The archeological shelter is asked to respond to several requirements:

- To cover an about $3.500 \mathrm{~m}^{2}$ surface without intermediate supports, and adjust to an irregular perimeter.

- To protect the archaeologists and the excavations from the weather.

- To provide an easy access to the visitors.

- To be simple to be assembled and disassembled also by not specialized handcraft.

- To be durable enough to remain in place for the whole duration of excavation.

\subsection{Proposed answer}

To respond to the specific demand, we retained a solution based on the reciprocal frame system. A built example of this structural system used for archeological purpose is the excavation shelter in Bibracte, France. It is an aluminum nexorade barrel vault shaped and covered with fabric, designed by (RFR and TESS) Gelez et al. [12].

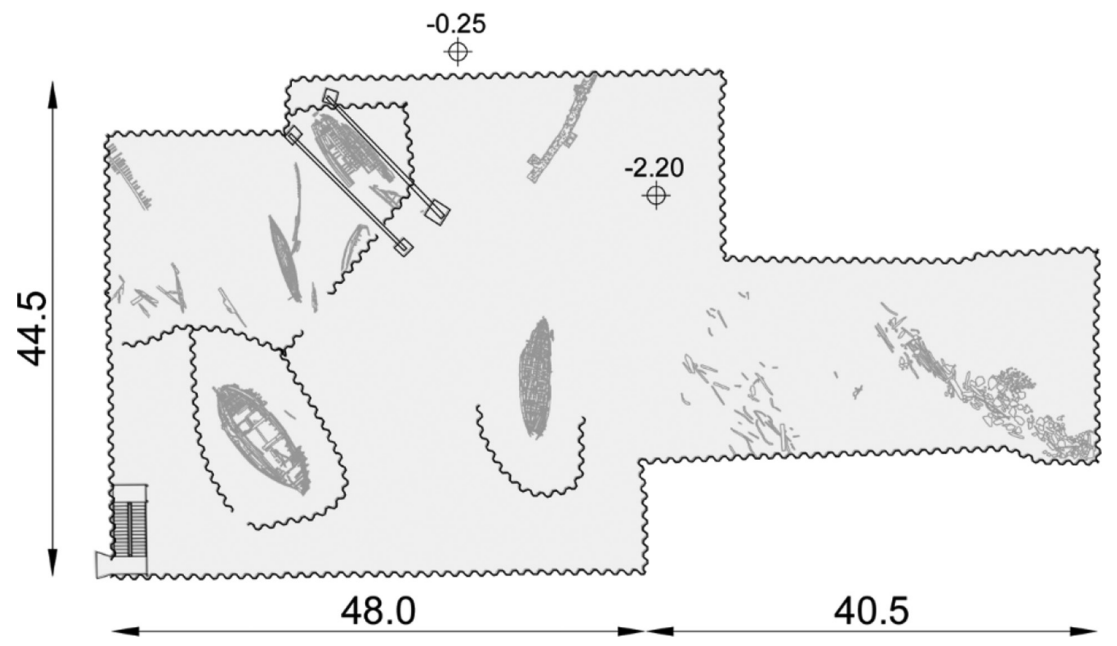

Figure 1: The archaeological site of the Roman Shipwrecks in Pisa. 
For our design purpose, we chose a timber nexorade shaped as a double curvature grid shell. Timber was selected as the building material because it allows for modular design and prefabrication, it is renewable and capable of satisfying the architectural demand. With simple product schedules, parts can be produced in different shapes and lengths using widely available fabrication tools, and assembled efficiently. Indeed, contrary to traditional grid shells, the connection between nexors can be achieved simply and quickly, without the needs of any sophisticated equipment. This feature, combined with the manageable size of structural elements and the relatively low requirement for scaffolding, makes this structure very suitable to satisfy the different requirements of a quick and temporary shelter of a large archeological area.

Aiming at optimizing both structural efficiency and esthetical qualities, the form finding process was oriented towards a funicular surface simply compressed under its self-weight. In that way, the shell could be supported only along the external perimeter, and cover the 45 meter-span excavation without intermediary supports. The shell presents openings along all the edges to allow visual continuity from the outside to the inside, along with necessary aeration (Fig. 2).

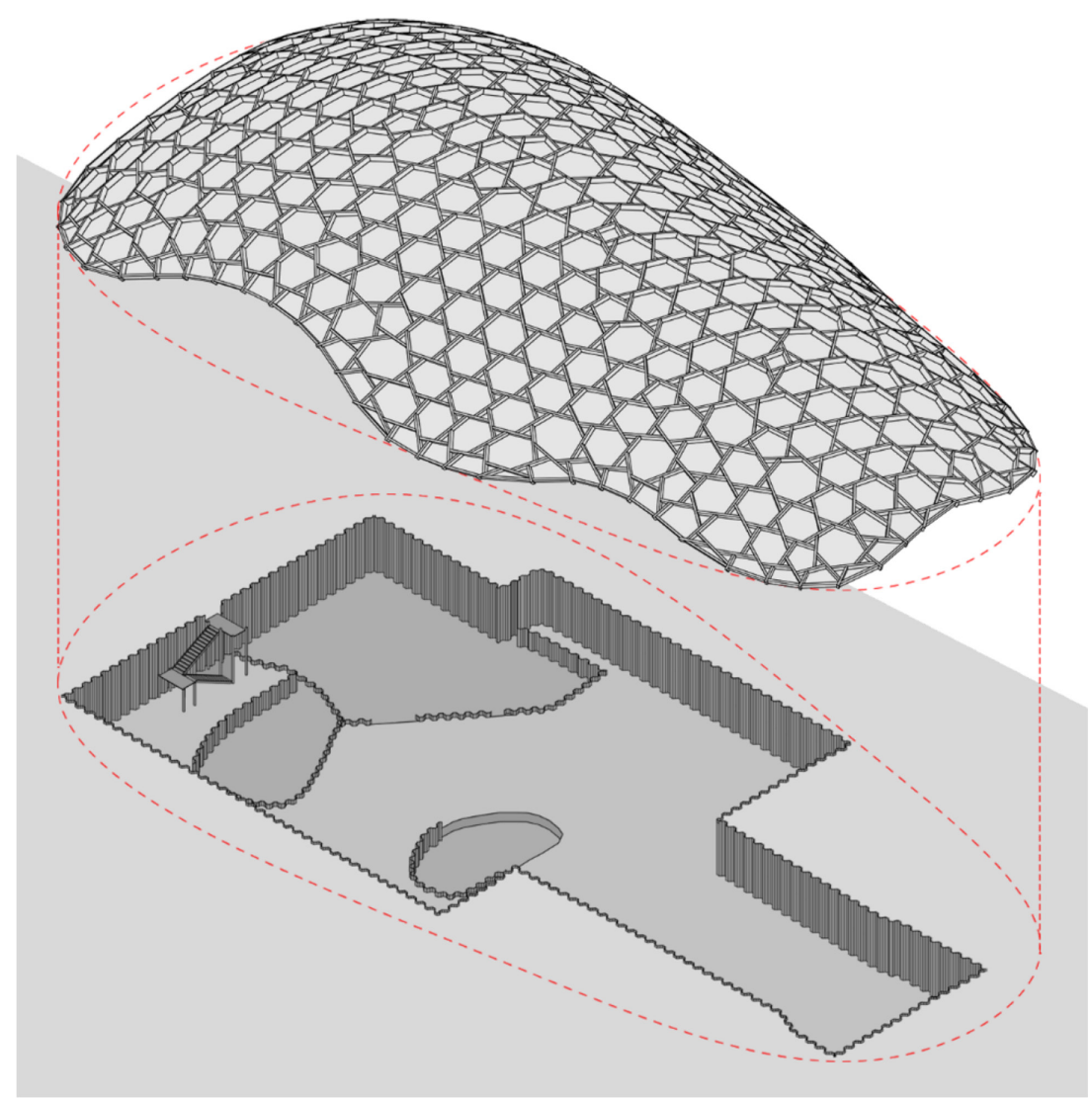

Figure 2: Design of the archeological shelter. 


\section{PARAMETRIC WORKFLOW}

\section{1 (Digital) form finding}

In architecture and structural engineering, form finding identifies the process of designing optimal structural shapes by using experimental tools and physical models to simulate a mechanical behavior. The oldest form-finding technique for shells is the reverse hanging model. A physical model, made with elastic cables or membranes, is first subject to gravitational forces to find out a structural state of pure tension; then it is inverted to obtain a compression-only surface [13]. Nowadays, physical models have been replaced by digital simulations that allow designers to investigate form in real time.

\subsection{Particle spring system}

One of the most popular digital forms finding techniques is the particle spring system, a discretization of a continuous model into a finite number of masses, called particles, connected by linear elastic springs. The particles are initially in a non-equilibrium state; when the simulation starts, they move until they reach an equilibrium position for a given set of parameters. For the form finding step, we used a physics based particle-spring system engine called Kangaroo [14], embedded directly within Rhinoceros ${ }^{\circledR}-$ Grasshopper. We found out a funicular shell using the following Kangaroo workflow [15].

- Boundary surface: A plane surface was created from a boundary curve approximating the shape of the site.

- Discretization: To simulate a deformable body, such as a cable net or a fabric membrane, a grid of springs must be defined. In order to create a grid, we converted the NURBS surface into a mesh, then we used Weaverbirds [16] to extract lines (mesh edges) and points (mesh vertices).

- Particle-spring system: After discretizing the geometry, lines were converted into springs and points into particles using Kangaroo. A uniform upward load was applied, so that the actual structure would have resisted vertical (gravity) loads. We anchored the membrane along its outline, by setting as anchor points the mesh naked vertices, i.e. vertices not bordered by faces, but leaving free the vertices along the openings.

- Kangaroo Engine: Particles, springs, forces and anchor points were finally connected to the Kangaroo Engine in their respective slots.

\subsection{Remeshing}

Since the continuous shell obtained through the particle spring system must be transformed into a grid shell, we converted the fine mesh in a coarse one. The mesh was then optimized in the following three steps.

- Hex-dominant remeshing: We employed a delaunay triangulation with Lloyd's relaxation to retrieve an initial hexagonal-dominant meshing. The meshing preserved the extrinsic simmetry of the input shape.

- Regularization: In order to uniform the length of mesh edges, we adopted local global shape optimization as proposed in [17]. This step improved the local shape of the faces of 

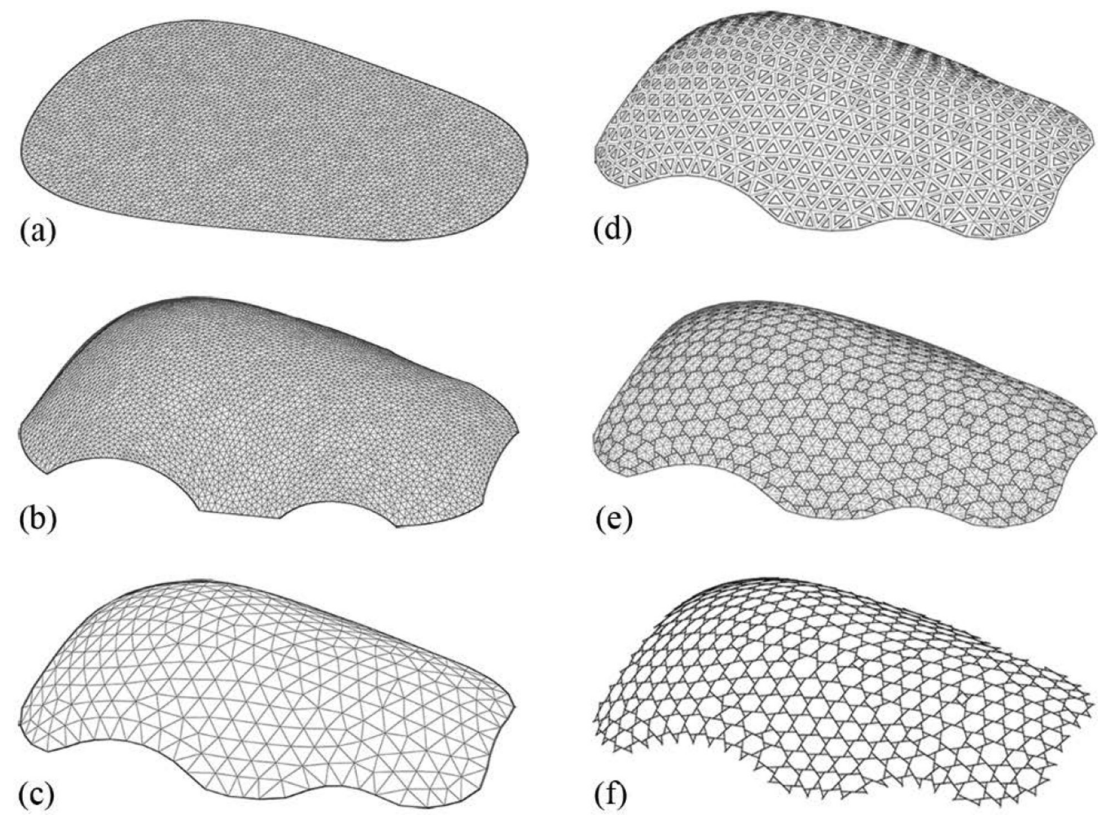

Figure 3: Parametric design workflow: (a) discretization; (b) particle spring system; (c) mesh optimization; (d, e) geometric construction of the grid; (f) dynamic relaxation.

our mesh, making them closer to the faces of Archimedeal solids; this geometric optimization phase greatly contributed to improve the aesthetics of the final grid shell.

- Triangulation: The last step consists of converting the hex-dominant mesh into a triangular one. This was obtained through a simple dualization step.

After the mesh optimization, we manipulated the elementary grid with multi-connected joints to generate the reciprocal grid with mono-lateral joints as follows.

- Geometric construction of the grid: First, the mesh edges were converted into polylines and scaled; then the mid points of the scaled polylines were connected to the midpoints of the original ones; finally, the obtained lines were extended.

- Dynamic relaxation: The members generated with the previous construction were straightened out using Kangaroo Engine.

- All the described workflow is shown in Fig. 3.

\section{STRUCTURAL ANALYSIS AND DESIGN}

\subsection{Structural model}

Starting from the defined RF grid, we set up the material as Glued Laminated Timber, and the cross section as $0.2 \times 0.6 \mathrm{~m}$. The beams are partially end-released for the bending moment component and the support joints are assigned to pinned connection. The material features refer to C24 with Service Class 3. The calculations are in accordance with the Eurocode [18]. The following loads were applied to the structure: dead-load of the structure, applied weight of the cladding, snow load and wind load. 
The structure own-weight is automatically calculated. Using the Karamba ${ }^{\circledR}$ "Meshload" component we transformed surface loads, applied to the initial mesh, into equivalent element-loads, applied to the nexors. Figure 4a shows the value of the axial stress obtained using first order theory.

\subsection{Buckling analysis}

Such as mostly compressed and lightweight structures, grid shells need to preserve a stable equilibrium configuration with respect to symmetric and asymmetric load cases. The importance of this aspect is due to the complex crisis modalities of such structures that is in some cases similar to the buckling of beams or the buckling of continuous shells, or in the worst scenario to a combination of both [19]. In general cases, the buckling analyses are conducted using FEM softwares, solving the generalized eigenvalue problem. Both geometric and material non-linearities as well as the presence of the imperfections should be considered. For the objectives of this work, we only performed geometrically non-linear analysis and we refer to further works for the introduction of imperfections. Figure $4 \mathrm{~b}$ shows the first buckling mode for the worst load case.

\subsection{Joint design}

A universal joint is a positive, mechanical connection between rotating shafts, used to transmit motion, power, or both. The simplest and most common type is called a Cardan joint. It
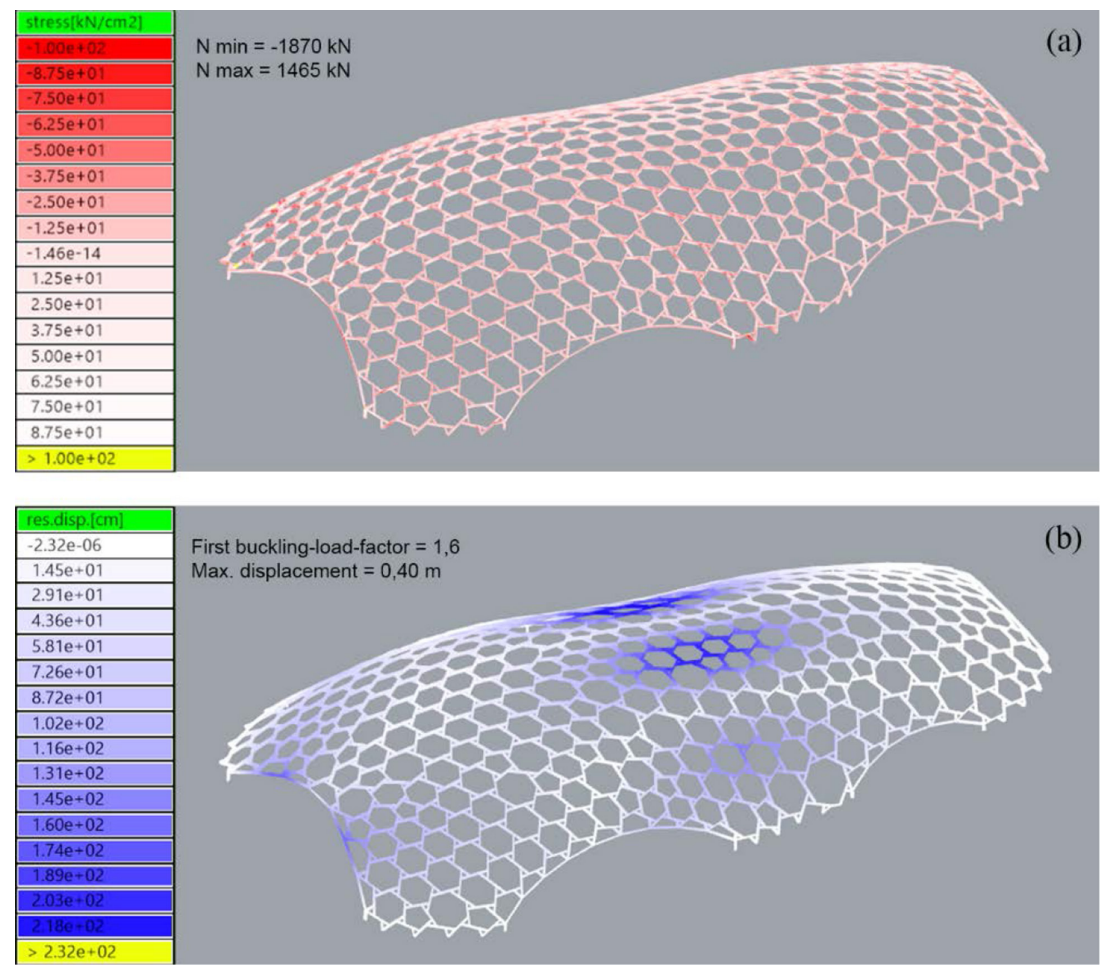

Figure 4: Structural analysis results: (a) axial stress, linear analysis; (b) first buckling mode, non-linear analysis. 

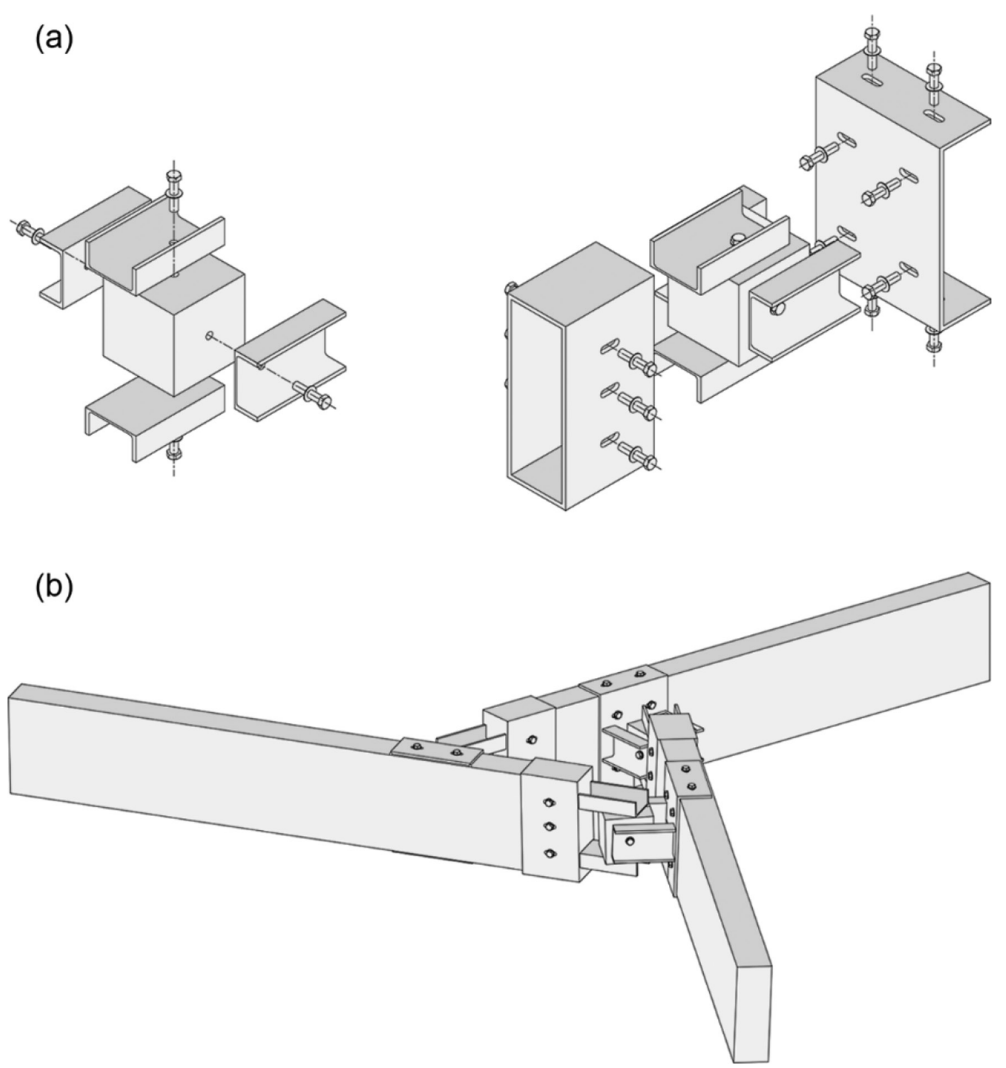

Figure 5: T-joinery system: (a) assembling phases; (b) base "fan" of the reciprocal grid.

consists of two yokes, one on each shaft, oriented at $90^{\circ}$ to each other and connected by a cross-shaped intermediate member called a spider. Inspired by the Cardan joint, the $T$ joinery system avoids any overlapping and axial eccentricities, but allows the necessary erection rotational and translational movements of accommodation (Fig. 5). The C-cross-section of the steel yokes increases the stiffness, and the slotted holes with friction bolts accommodate the length imperfections due to the fabricate and assembly process. The spigot joints are efficient because the shell structure is mainly compressed. Indeed, a confined wooden connection achieves a better structural behavior.

\section{DIGITAL FABRICATION}

A model using CNC laser-cutting will be constructed to experiment the assembly sequence and test the structural feasibility of such a design approach. Before creating the final model, we tested the tolerances realizing a hemispherical model made of 64 pieces, shown in Fig. 6. Tenon mortise joints are used to accelerate the model assembly, even if they don't allow the same rotational freedom of the joints intended for use in the full-scale structure. The digital model was created through the following workflow.

- Associative rule of elements: We found out which and where other elements are connected to the former. 
(a)

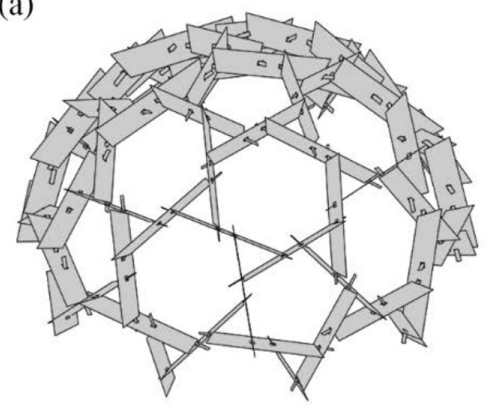

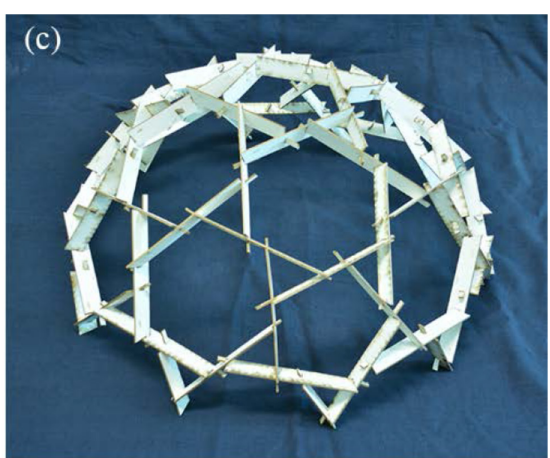

(b)

\begin{tabular}{|c|c|c|c|c|c|c|c|c|c|c|c|c|c|c|c|c|}
\hline 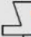 & 028 & 627 & 0 & 628 & $\sqrt{020}$ & $\mathrm{~b}^{0}{ }^{0}$ & $b_{30}$ & 629 & 030 & 639 & 0 & b30 & $\sqrt{\text { a31 }}$ & 628 & 0 & b317 \\
\hline 당 & .24 & bs 90 & $0_{032}$ & b24 & $\int_{0.25}$ & ${ }_{021}^{0}$ & ${ }_{026}$ & ${ }^{b 25} L$ & J.26 & 0 & $\mathrm{~B}_{32}$ & b26 & .27 & $65^{\circ}$ & $a_{b 2}$ & b27 \\
\hline 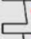 & 020 & $618^{\circ}$ & ${ }^{0}{ }_{634}$ & 620 & a.21 & ${ }_{654}{ }^{\circ}$ & $\mathrm{O}_{\mathrm{b} 22}$ & b21 & 0.22 & $.30^{\circ}$ & $0_{0.58}$ & ${ }^{b 22} L$ & .023 & ${ }_{44}^{\circ}$ & $0_{024}$ & $b_{223}$ \\
\hline ᄃ & alo & $.017^{0}$ & $0{ }^{0}{ }_{027}$ & blo & a17 & $.15^{0}$ & ${ }^{0}{ }_{b 50}$ & b17 & 018 & $043^{\circ}$ & $0_{019}$ & b18 & 019 & $.20^{\circ}$ & ${ }^{0}{ }_{052}$ & b19 2 \\
\hline 다 & 012 & $613^{\circ}$ & 0 & b12 & a13 & $649^{\circ}$ & $O_{b 14}$ & b13 L & o14 & $40^{\circ}$ & ${ }_{0}{ }_{a 12}$ & \begin{tabular}{l|l} 
b14 & . \\
\end{tabular} & o15 & $0^{\circ}$ & & b15 \\
\hline ᄃ & 08 & $69^{\circ}$ & $0_{042}$ & 68 & 99 & $.040^{\circ}$ & $\mathrm{O}_{056}$ & bo $L$ & 010 & $5^{\circ}$ & ${ }^{0}{ }_{\text {all }}$ & b10 & all & 6570 & 0 & bा1 $\angle$ \\
\hline ᄃ & 04 & $05^{\circ}$ & ${ }^{0}{ }_{031}$ & $b 4$ & 05 & $63^{\circ}$ & ${ }_{b 20}$ & b5 & 06 & ${ }_{0} .55^{\circ}$ & 0 & b6 & 07 & $.00^{\circ}$ & 0 & b7 \\
\hline$J$ & 00 & ${ }^{0}$ & $0_{620}$ & bo & of & $625^{\circ}$ & ${ }_{0}{ }_{02}$ & bा & 02 & $0^{\circ}$ & $0^{0}$ & $b 2$ & 03 & ${ }_{0.33}^{\circ}$ & $0_{04}$ & $\begin{array}{ll}b_{3} \\
\end{array}$ \\
\hline
\end{tabular}

Figure 6: Digital fabrication: (a) digital model; (b) pieces are oriented on a plane, nested and labelled; (c) assembled model.

- Creation of pieces: After assigning the cross section to the RF elements, Tenon was created by adding two slaps at the edges of each element, mortises by holing each element in the right positions.

- Packing elements: The elements were sectioned with a middle plan, then the section surfaces were oriented on the XY plane and nested to minimize the material waste.

- Assembly instruction: We assigned a number $\mathrm{x}$ to each element, labelling its two end slaps as "ax" and "bx". Thanks to the found associative rules, the same label was printed on the slap and the hole of each Tenon and mortise joint to simplify the model assembling.

\section{CONCLUSIONS}

The RF system was successfully applied to the design of a large-spanned, reversible, timber grid-shell, and well satisfied the specific requirements of the archeological shelter of the Roman Shipwrecks in Pisa. A good structural response was achieved by taking into account static behavior since the first design phases.

The workflow is completely parametric and allow to design RF structures with different initial geometries and patterns. Indeed, starting from the 3D configuration allows a better control on the final shape compared to mapping a pattern into a surface. The beams have different length, but they can be easily produced through digital fabrication. Moreover, the cardan joint can accommodate some imperfections of the process, and also the axial eccentricity between beams within an acceptable domain. As universal, the joinery system allows to quickly and easily assemble and disassemble the shelter, and to re-use the connections in a different configuration. The CNC-cut test model proved to be very stiff and easy to build. Thanks to labels printed on pieces, and knowing that the fans are clockwise oriented, it was 
assembled without using any drawings. A further scheduled work regards the construction of a physical model of the grid shell, made of more than one thousand pieces and spanning for 2 meters.

Numerical future work will be oriented towards a deeper structural analysis, by exporting the model to FEM softwares to check the results from Karamba®, and to introduce the imperfections in the geometric non-linear analysis. Another aim is to develop a cladding system for the grid shell. Most times, realized RF structures are cladded by membrane materials such as fiber-reinforced PVC sheet. A paneling option is complicated by the lack of planar openings in the grid. The problem could be solved through a cladding system made of polygonal shingles, inspired to the skins of some reptiles. This cladding system would allow the natural aeration and waterproofing. Above all, it would be possible to have planar or singular curvature panels, considerably reducing the fabrication costs. The development of this panel tectonic would add immensely to the applicability of the reciprocal frame to complex architectural geometry.

\section{REFERENCES}

[1] Popovic Larsen, O., Reciprocal Frame Architecture, Elsevier, 2008.

[2] Tamke, M., Riiber, J. \& Jungjohann, H., Generated Lamella, LIFE in: formation. On responsive information and variations in architecture, New York, ACADIA, pp. 340-347, 2010.

[3] Nabaei, S.S. \& Weinand, Y., Geometrical description and structural analysis of a modular timber structure. International Journal of Space Structures, 26(4), pp. 321-330, 2011. http://dx.doi.org/10.1260/0266-3511.26.4.321

[4] Baverel, O., Nooshin, H. \& Kuroiwa, Y., Configuration processing of nexorades using genetic algorithms. Journal of the I.A.S.S, 45(2), pp. 99-108, 2004.

[5] Douthe, C. \& Baverel., O., Design of reciprocal frame systems with the dynamic relaxation method. Computers \& Structures, 87(21-22), pp. 1296-1307, 2009. http://dx.doi.org/10.1016/j.compstruc.2009.06.011

[6] Kohlhammer, T. \& Kotnik, T., Discrete analysis - a method to determine the internal forces of lattices. International Conference on Space Structures, London, 2011.

[7] Kohlhammer, T. \& Kotnik, T., Sistemic behaviour of plane reciprocal frame structures. Structural Engineering International, 21(1), pp. 80-86, 2011. http://dx.doi.org/10.2749/101686611X12910257102596

[8] Song, P., Fu, C., Goswami, P., Jianmin Z., Mitra, N., J. \& Cohen-Or, D., Reciprocal frame structures made easy. ACM Transactions on Graphics, 32(4), 2013. http://dx.doi.org/10.1145/2461912.2461915

[9] Rutten, D., Grasshopper: generative modeling for Rhino, Computer software, 2012, available at: http://www.grasshopper3d.com

[10] McNeel, R., Rhinoceros: NURBS modeling for Windows, Computer software, 2010, available at: http://www.rhino3d.com

[11] Preisinger, C., Karamba: parametric structural modeling, Computer software, 2011, available at: http://www.karamba3d.com

[12] Gelez, S., Aubry, S. \& Vaudeville, B., Nexorade or Reciprocal Frame System Applied to the Design and Construc on of a $850 \mathrm{~m}^{2}$ Archaeological Shelter. International Journal of Space Structures, 26(4), pp. 303-311, 2011.

http://dx.doi.org/10.1260/0266-3511.26.4.303 
[13] Adriaenssens, S., Block, P., Veenendaal, D. \& Williams, C., Shell Structures for Architecture: Form Finding and Optimization, London, 2014.

[14] Piker, D., Kangaroo: Live Physic Engine, Computer software, available at: http:// kangaroo3d.com

[15] Tedeschi, A., AAD _ Algorithm Aided Design, Parametric strategies using Grasshopper, Le Penseur, Brienza (Potenza), Italy, 2014.

[16] Piacentino, G., Weaverbird: topological mesh editor, Computer software, available at: http://www.giuliopiacentino.com/weaverbird/

[17] Pietroni, N., Tonelli, D., Puppo, E., Froli, M., Scopigno, R. \& Cignoni, P., Statics aware grid shells. Computer Graphics Forum, 34(2), pp. 627-641, 2015. http://dx.doi.org/10.1111/cgf.12590

[18] UNI ENV 1991-2, Eurocode 1, Basis design and actions on structures, 1997.

[19] Knippers, J. \& Bulenda, T., Stability of grid shells. Computer \& Structure, 79(12), pp. 1161-1174, 2001.

http://dx.doi.org/10.1016/S0045-7949(01)00011-6 